\title{
Reconstruction of the protective barrier on the highways in the republic of North Macedonia pursuant to the MKS en1317 standard
}

\author{
Riste Ristov ${ }^{1,}{ }^{*}$, Slobodan Ognjenovic ${ }^{2}$, Ivana Nedevska $^{3}$, and Zlatko Zafirovski ${ }^{4}$ \\ ${ }^{1}$ PROSTOR DOO Kumanovo, RNM, North Macedonia \\ ${ }^{2}$ Faculty of Civil Engineering, Skopje, RNM, North Macedonia \\ ${ }^{3}$ Eskavatori-MK DOOEL, Skopje, RNM \\ ${ }^{4}$ Faculty of Civil Engineering, Skopje, RNM, North Macedonia
}

\begin{abstract}
The purpose of this paper is to offer an approach to elaboration of technical documentationon the reconstruction of tghe existing protective barriers, as pursuant to the MKS EN 1317 standard, on all the roads in the Republic of North Macedonia. The current situation regarding the protective barriers on the motorways is the following: short protective lengths (shorter than the tested one), unprotected obstacles (non-standards boards, portals, road lamps, concrete pillars, tunnel portals, logs etc.), protection of third persons is not taken into consideration, Inappropriate application of the U.S4 standard (use of aluminum instead of steel profiles, barriers of metal sheets thinner than the standard ones, inappropriate pillars, bolts of lower quality than the prescribed one, larger distance between pillars, thinner anti-corrosion layer etc.) The new EN1317 standard dictates complete coverage of all the above cited shortcomings, regardless of whether positions that do not exist in the previous standard or omissions upon designing or performance are considered. The paper shall present the actual problems identified upon the design with permanent protection and their elimination with the new standard of MKS EN 1317.
\end{abstract}

\section{Introduction}

Upon the identification of the current condition of the highways it was concluded that the existing barrier was in a quite bad shape, without the necessary retention system, it is not properly constructed, is damaged and sometimes not sufficiently long. Not all dangerous areas are protected, as are the grid girders of traffic boards, portal girders pf traffic signalization, light poles, big and open gutters, unsafe finishings towards the New Jersey concrete barrier. Front parts of retaining walls built over the pavement, high and steep embankments, pay tolls etc.

Besides the mentioned problems related to the existing barrier, there are, on the particular road section, problems with the placement of the new vehicle retention system. There are locations where it is not possible to place a protective elastic barrier due to lack

\footnotetext{
* Corresponding author: risteristov333@yahoo.com
} 
of an appropriate base (bridges, overpaqsses, culverts, entry heads of culverts etc.) as pursuant to the EN 1317 standard. Due to this, there are sections where it is not rational to place a vehicle retention system (elastic protective barrier) because it is necessary to perform certain preparatory works ( e.-g elimination of self-growing trees, of the vegetation, replacement of inappropriate curves, repair of retaining walls, reconstruction of big concrete culvert heads etc.) to eliminate the need of a protective barrier), In order to place an appropriate vehicle retention system, it is also necessary to previously relocate or replace certain traffic signalization elements, as are certain grid girders of non-standard boards, relocation of the lighting, reconstruction of the concrete paths for inspection of constructions, reconstruction of big concrete manholes, repair of retaining walls, rock slopes etc.

\section{Problems necessitating civil engineering activities}

\subsection{Bridges, underpasses and overpasses}

On most constructions there are no necessary conditions for placement of a protective barrier. concrete paths for maintenance are not performed with the sufficient width to host a protective barrier with the necessary level of retention and the necessary dynamic slope, and in some constructions the concrete is a very bad condition.

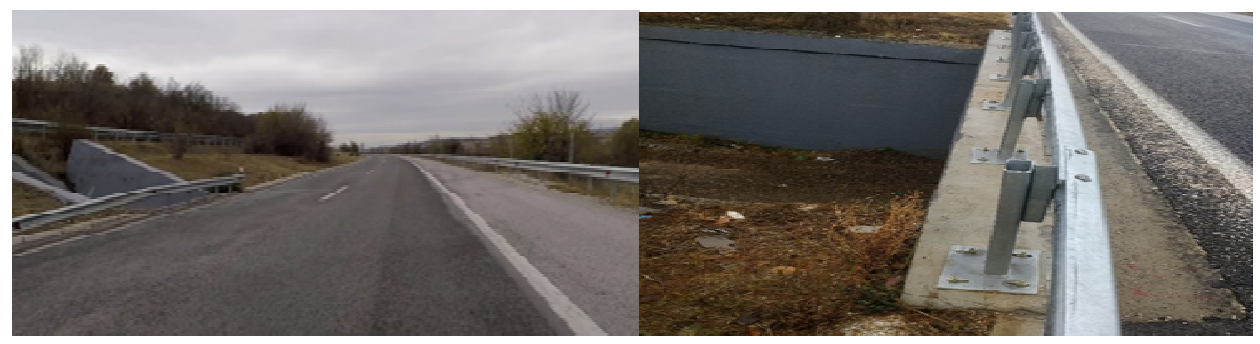

Fig. 1. Underpass at the Kumanovo-Miladinovci section A1(E75), km 20+112.

One of the possible solutions is the reconstruction (renewal and expansion) of the concrete pagths for maintenance of the constructions, thereby providing for conditions of placement a H2-W4 protective barrier (in constructions for protection of third persons) or a H1-W2 (smaller constructions where no third persons are endangered). The maintenance paths should be separated from the pavement by a curb not higher than $7.0 \mathrm{~m}$.

In case of smaller culverts, it is necessary to construct a concrete beam to provide for conditions to place the protective barrier (in constructions for protection of third people) or H1-W2 (smaller constructions not endangering third people).

\subsection{Concrete (entrance) heads of pipe culverts in excavation}

The bid concrete entrance heads are built of massive concrete higher than the pavement and placed by its edge, which is a massive non-deformable pbstacle. 


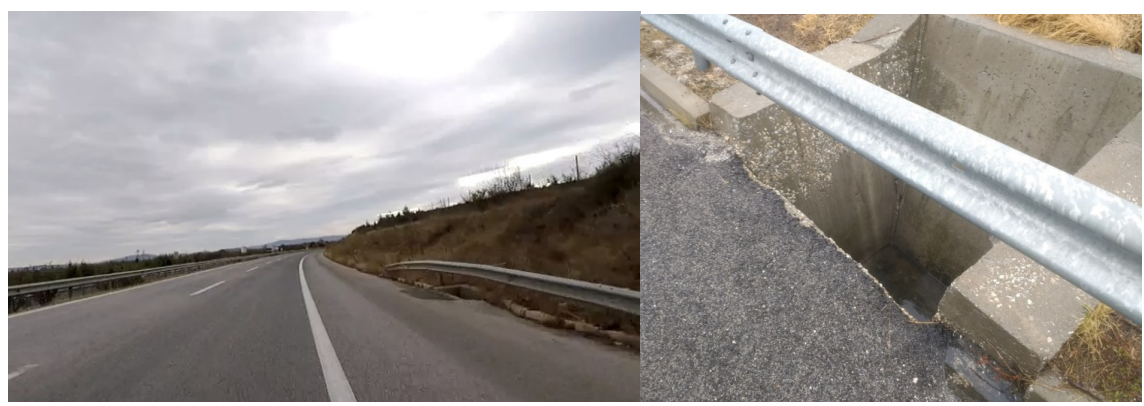

Fig. 2. Entrance head of a pipe culvert A1(E75), km 15+830 (west pavement lave.

These obstacles can be protected by a $\mathrm{H} 1$ protective barrier. A possible alternative is the reconstruction of an entrance head and its reduction to the pavement level, covering it with a steel grid on the top, without any need to place a protective barrier.

\subsection{Retaining walls beneath the pavement}

In most cases, the crown of the retaining walls is over the levelling of the pavement and is a massive and non-deformable obstacle. In such cases the placement of an elastic protective barrier in the retaining wall is a problem as the width is not sufficient to host the barrier between the pavement edge and the wall crown.

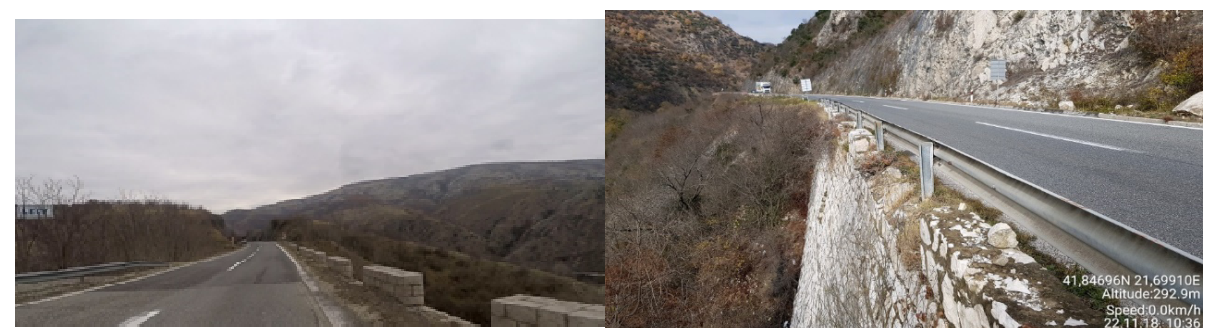

Fig. 3. A crown elevated from a retaining wall A1(E75), km 62+930 and km 47+480.

Some possible solutions are:

- Reduction of the width of the existing pavement (stopping lane) and placement of the barrier between the crown and the edge of the pavement, or

- Destruction of part of the wall and construction of a new crown at the pavement level and placement of a protective beam.

Only the second solution can be applied on the road sections where retaining walls with a crown have been constructed over the pavement level, where there is no possibility to narrow the lanes and no lane for forced stopping.

\subsection{Steep slopes in cuts and notches of the road}

The steep and rigid slopes in the cuts and notches on the road are a big problem, as they are very near to the edge of the pavement. . There is no berm on which it is possible to place a protective barrier. Additional danger are the frequent landslides on that side of the road. 


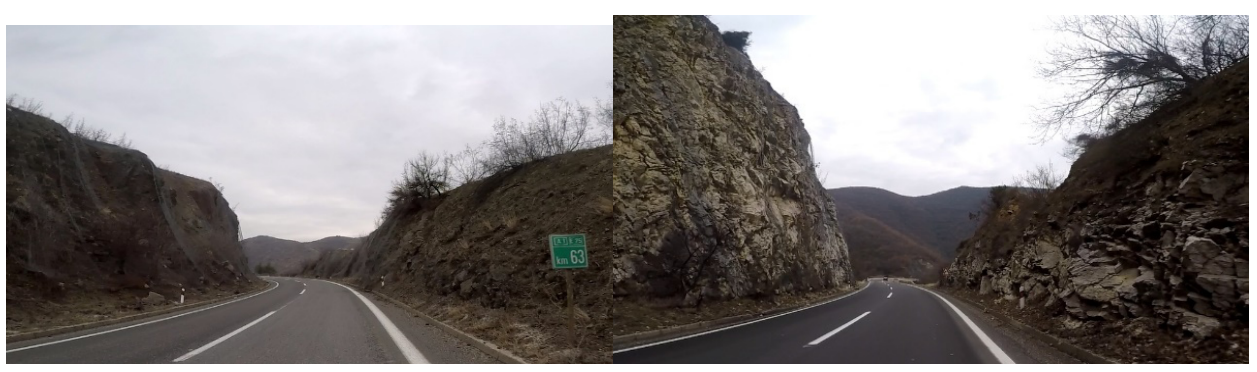

Fig. 4. Steep rock slopes A1(E75), km 63+000 and km 42+000.

The solution is to destroy and form the slopes to enlarge the berm on which a protective barrier can be placed. The formation and stabilization of slopes will reduce the possibility of landslidesThis solution is extremely expensive due to a large number of such moves and earth works.

\subsection{Narrow shoulders}

On certain sections, where it is necessary to place a protective barrier, a problem I created by the insufficient width of the shoulder.

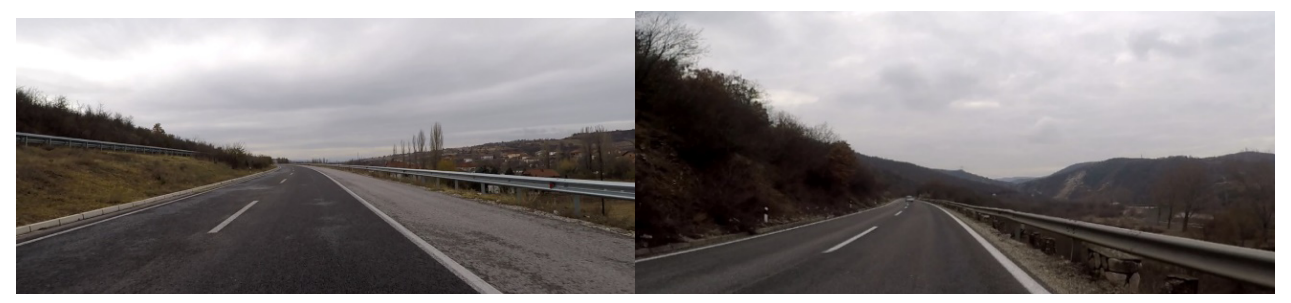

Fig. 5. Narrow shoulders A1(E75), km 24+200 and km 43+400.

Several solutions are possible upon such cases:

- Recovery by forming a shoulder of at least $1.5 \mathrm{~m}$ wide enough to construct the protective barrier of H1-W5,

- Placement of a H1 protective barrier H1 with smaller impact area (W2, W3 and W4).

- Where neither of the two solutions is possible, a concrete foundation should be constructed on which the barrier should be placed. Before that it is necessary to remove the stone walls higher than the pavement [1].

\section{Problems for which convil engineering activities are unencessary}

\subsection{High curbs}

On certain sections of the route there are high curbs also on the right-hand side of the pavement (next to the lane for forced stopping or to the driving lane if no lane for forced stopping exists) The placement of the barrier in line with the edge of the pavement can in such circumstances be very close to the vehicles moving along the pavement, which has a negative influence on traffic safety. 


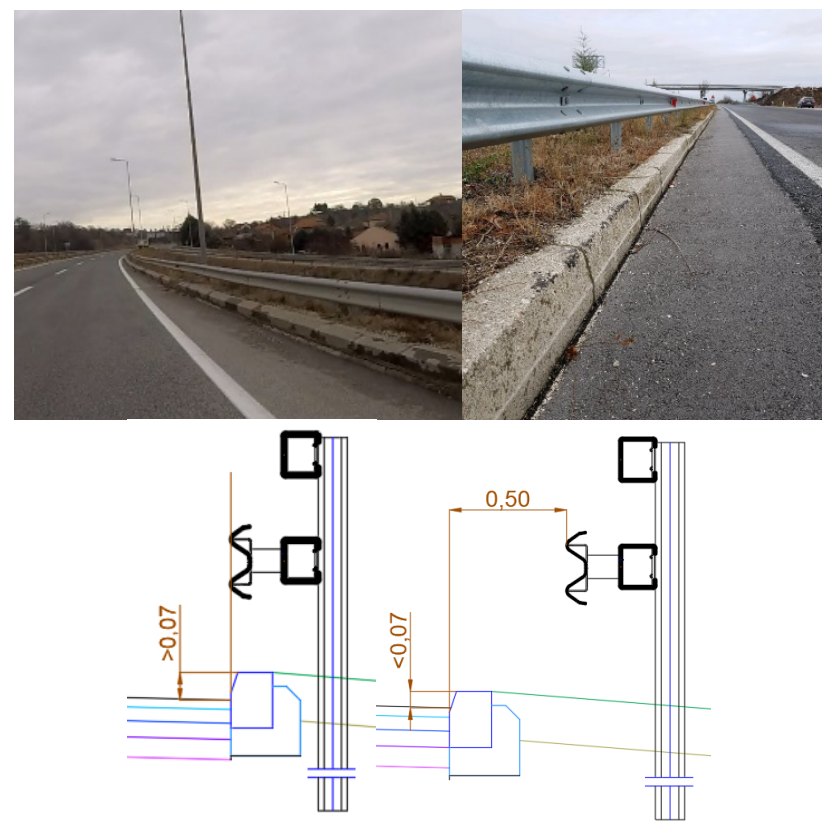

Fig. 6. High curves A1(E75), km 26+400 and km 3+400.

Two solutions are recommendable in such cases:

- Placement of the barrier in line with the curve or,

- Replacement of the existing curbs by new ones $7 \mathrm{~cm}$ high and construction of a protective barrier at $0.5 \mathrm{~m}$ from the edge of the pavement.

The first solution, the placement of the barrier in line with the curb, can be applied only on the right-hand side of the pavement when there is a traffic lane for forced stopping or next to the overpassing lane of there is a flume alongside the pavement.

The second solution, replacement of the existing curbs with new ones up to $7 \mathrm{~cm}$ high and the placement of a protective barrier at 0.5 from the edge of the pavement should be applied when the barrier is placed next to the overtaking lane or to the driving lane, if there is no lane for forced stopping where the flume next to the pavement has not been constructed.

\subsection{Trees along the pavement and the verge}

Occasionally, there are trees along the whole particular section along both sides of the pavement and the verge.

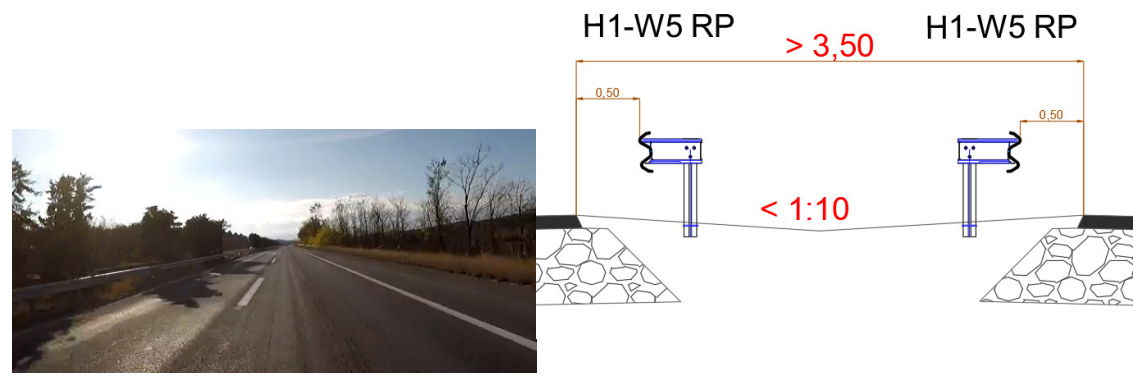

Fig. 7. Trees on the division belt and along the pavement A1(E75), km 105+550. 
Possible solutions:

- The trees along both sides of the pavement should be protected by an H1 barrier. If the pavement lanes are in the same level, an $\mathrm{H} 1$ barrier can be placed along the verge for both directions which, by its joining effect provides for an $\mathrm{H} 2$ protection level (for which the appropriate impact tests will of course be necessary). If there is any obstacle along the verge, the common effect of the $\mathrm{H} 1$ barrier cannot be achieved, and a $\mathrm{H} 2$ barrier should be placed on both sides (for the both directions) of the verge.

- Removal of the trees from both sides of the pavement and in the verge as well.

As it is mentioned above, the second solution is the better one, as there will be no need to remove the $\mathrm{H} 2$ barrier at the largest part of the route on both sides of the pavement, but a tandem barrier would be placed with the H1 protection level, which is certainly amore economical solution. But such a solution of tree-removal would most probably be impossible due to restrictions conditioned by environmental requirements.

\subsection{Advertizing billboards and totems}

The advertising billboards and totems are massive no-deformable roadside obstacles.

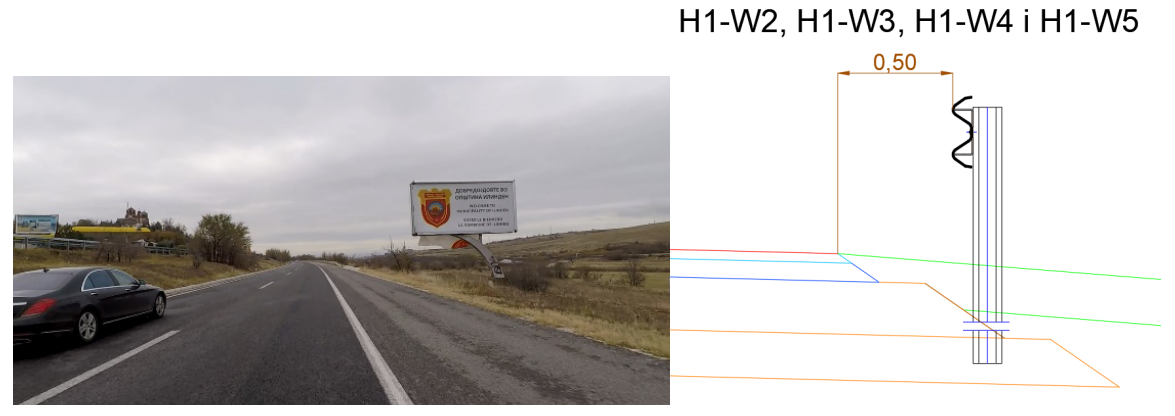

Fig. 8. A totem and a billboard A1(E75), km 24+800.

Possible solutions:

- Removal or relocation of ilboards and totems.

- H1 protective barrier.

The most acceptable solution is the second one, i.-e the construction of a protective barrier.

\subsection{Impact buffer (absorber)}

On the exit locations on intersections, at gas stations, at lay-by locations or at the beginning of the verge there are massive non-deformable obstacles dangerous to third persons.
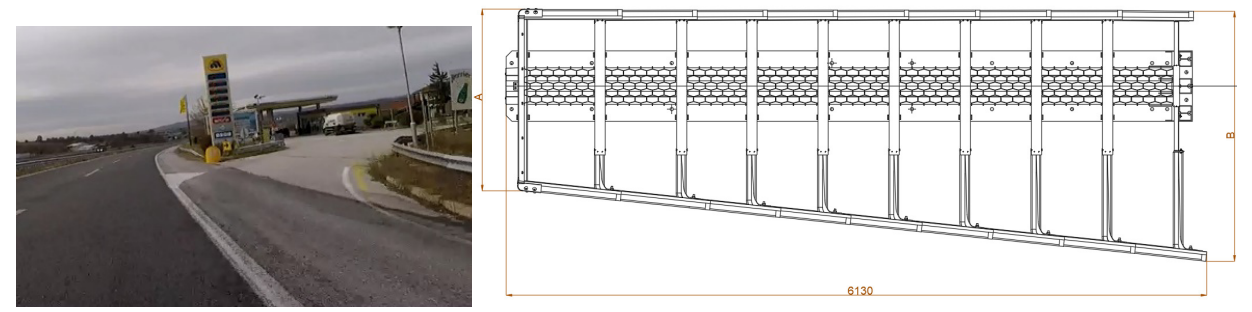

Fig. 9. Impact buffer A1(E75), km 18+55. 
These massive obstacles and the area risky to third persons should be specuially protected. The solution to these and similar problems is to install impact buffers at te beginning of tghe protective barrier.

\subsection{Entry into a tunnel}

The tunnel entries are usually unprotected, running the risk of a direct collision with the vehicle in the tunnel's entry portal.
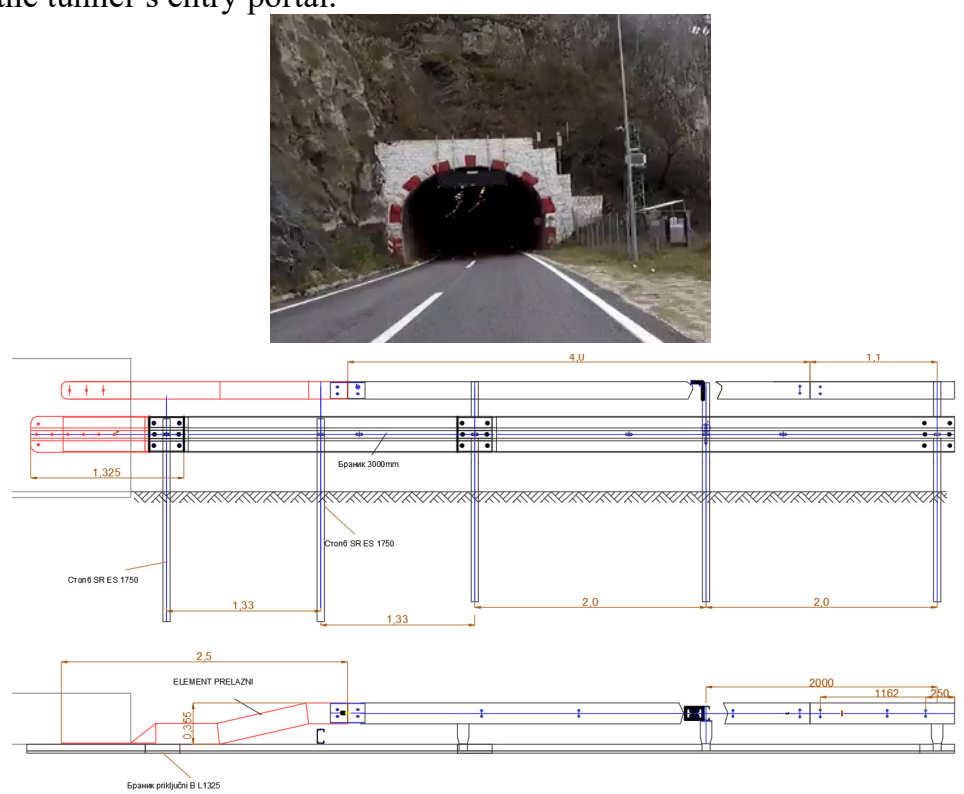

Fig.10. Entry into a tunnel A1(E75), km 47+150.

Placement of an $\mathrm{H} 2$ protective barrier over the tunnel on both sides of the pavement and fitting the entry portal of the tunnel with the transitory element of the barrier.

\subsection{Traffic signs with braced girders beside the pavement}

Many traffic signs on braced girders are placed near the pavement. Some traffic signs should be protected by barriers with a higher protection capacity, which are more expensive. Certain traffic signs are so close to the pavement that it is impossible to place any barrier between them and the pavement.

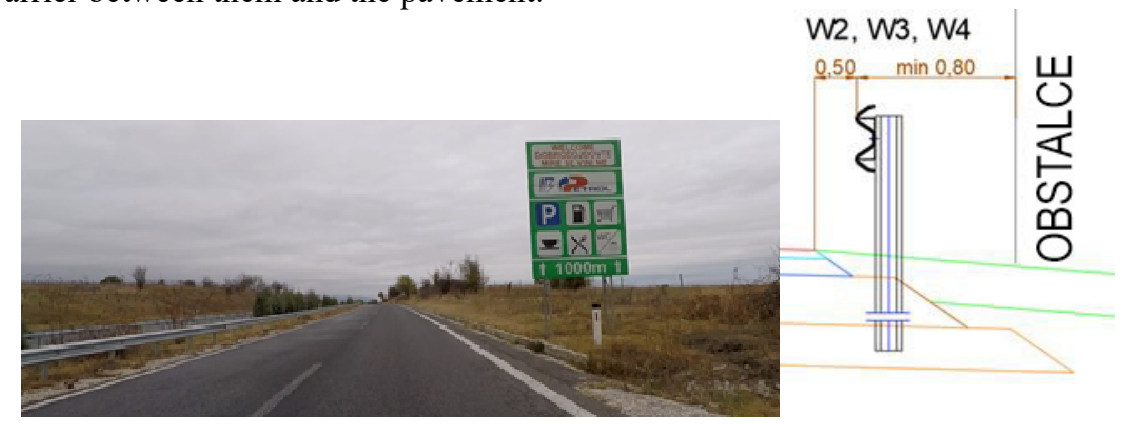

Fig. 11. A non-standard traffic sign A1(E75), km 16+220. 
Possible solutions:

- Placement of a barrier with a smaller area of action (W2, W3 and W4).

- All signs placed on braced girders should be relocated at $2.7 \mathrm{~m}$ from the edge of the pavement or $1.7 \mathrm{~m}$ from the flumes, and the H1-W5 barrier should be placed respecting the necessary length before and after the traffic sign.

Considering all costs, especially those for relocation of the traffic signs, for the placement of a new barrier and the potential barrier maintenance costs, and the fact that it is not even possible to place a barrier near some traffic signs, the second solution proves itself as the only acceptable one.

\subsection{Bridges with a concrete barrier (New Jersey)}

Many bridges have been constructed featuring solid concrete barriers. The problem is to place the protective barrier before and after the bridge and its fitting into the concrete barrier of the bridge.
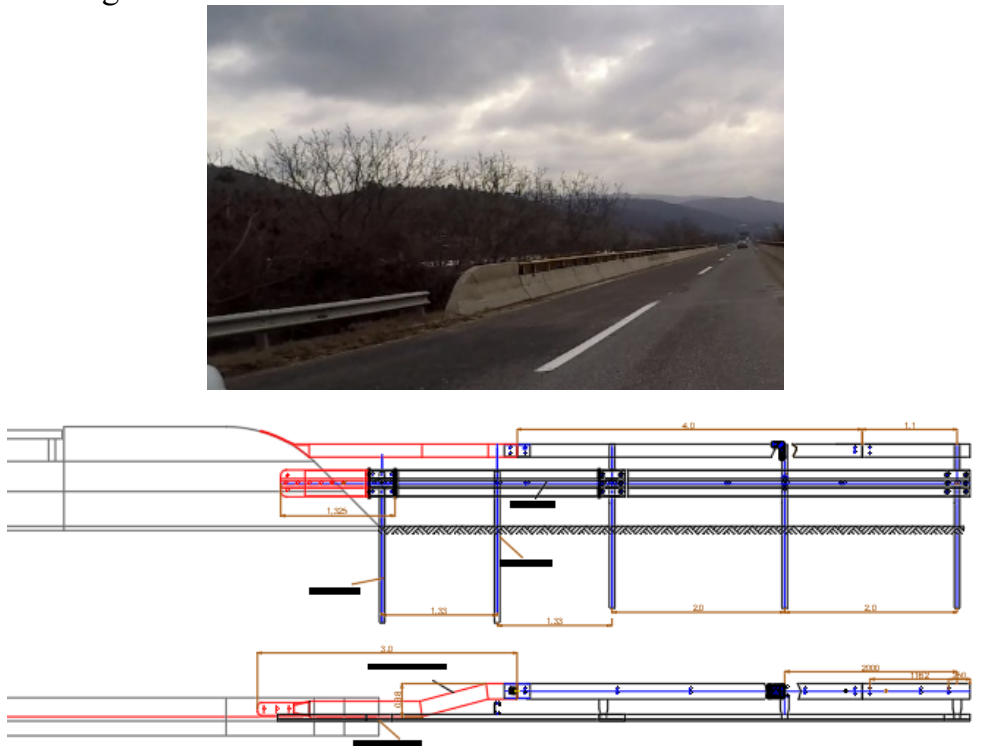

Fig. 12. Concrete barrier on a bridge A1(E75), km 42+900.

Possible solutions:

- The concrete barriers should be removed from the bridge, new concrete maintenance paths should be built; also an elastic protective barrier with the appropriate retaining level should be installed.

- Before the brighr it is necessary to install a barrier with a retaining level of $\mathrm{H} 2$ and the concrete barrier is to be fitted in by transitory elements.

The optimal solution to this problem with the least financial implications is the second variant.

\subsection{Retaining walls over the pavement}

The retaining walls over the pavement are a massive non-deformable obstacle. 


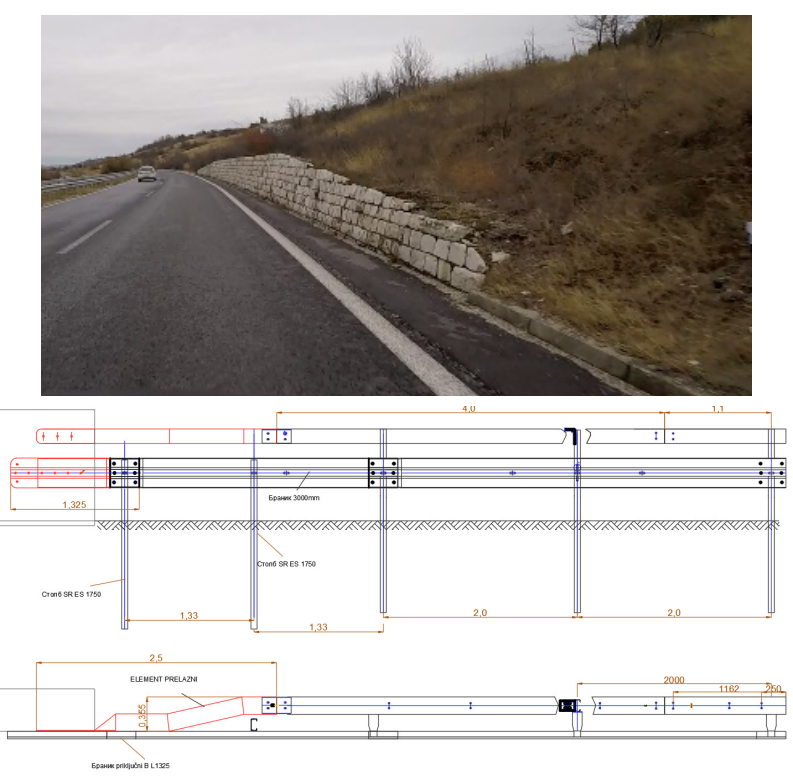

Fig. 13. Retaining wall A1(E75), km 23+850.

In these cases the $\mathrm{H} 2$ barrier should be installed in front of the retaining wall. Transitory elements are necessary for the retaining wall to fit in.

\subsection{Traffic sign portals}

The portal bearers are an obstacle that can put people in danger. The problem is that some portal pillars and concrete foundations are too near to the edge of the pavement or along that verge, which makes it physically impossible to place the necessary barriers.

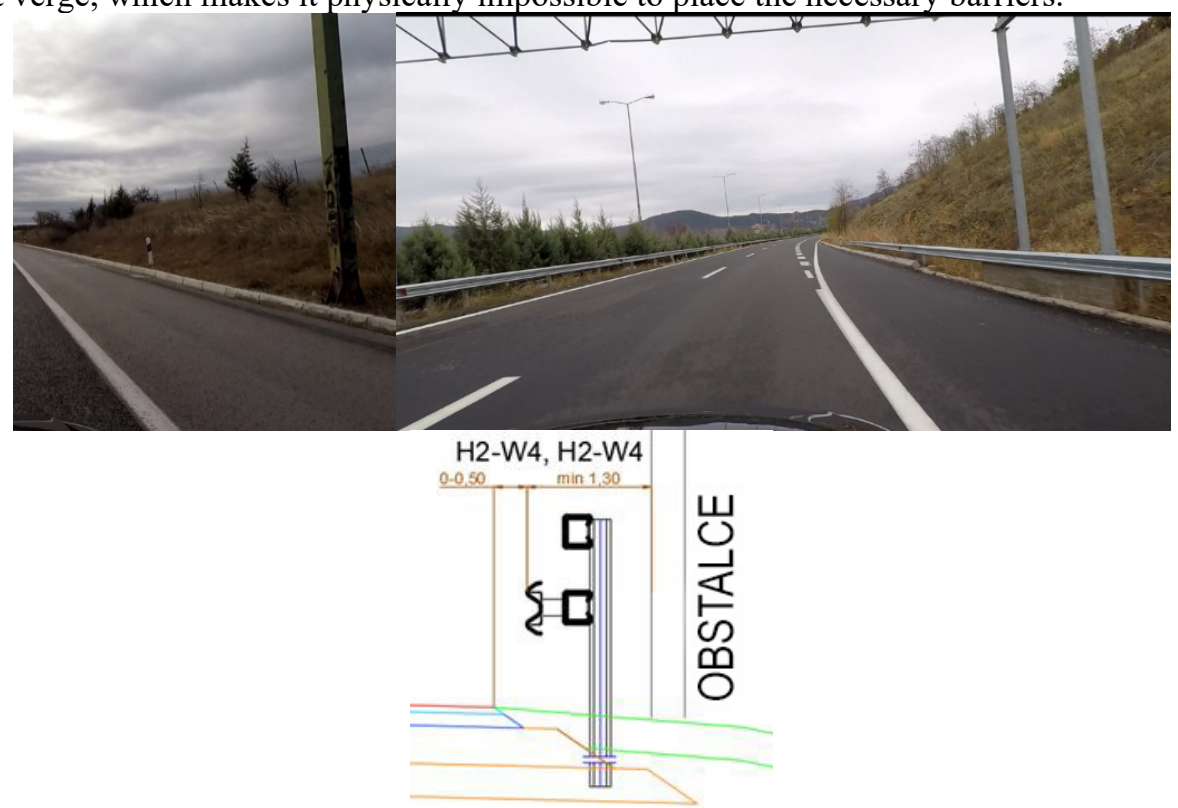

Fig. 14. Portal A1(E75), km 8+400 and km 126+100. 
It is necessary to place am $\mathrm{H} 2$ protective barrier at these portal pillars. To achieve this, some portal pillars should be moved off.

\subsection{Lighting poles}

Lighting poles also represent and underfomable obstacle. The problem is that certain poles are near to the edge of the pavement, due to which a barrier ensuring better protection is to be placed, in some cases even close to the edge of the pavement.

The H1 barrier is to be placed in lighting poles except in cases close to the edge of the pavement. In this case the barrier is to be placed nearer to the edge of the pavement [1].

\section{Design solution}

The design solution is in full compliance with the EN 1317 standard, as well as with the technical Instructions on the application of road restraint systems in Republic of North Macedonia, issued by the State Roads Public Company in 2018, which complies with this standard.

The design envisages the installation of a protective barrier H1W2-W5 and H2W4 on a shoulder, H1W2 and H2W4 on a construction and transitory elements between the barriers $\mathrm{H} 1$ and $\mathrm{H} 2$. Wherever it is allowed by the field conditions, the barrier installed $60 \mathrm{~m}$ before and 30 over dangerous spots. The said length does not include the $12 \mathrm{~m}$ long initial and final constructions [2].

\section{Conclusion}

The considerable shortcomings in highway traffic safety were eliminated through the applied solutions emphasizing the needs of their upgrading. This project only solved the actual situation and only the current problems. A little shortcoming in this design documentation is that the upcoming traffic solutions were not taken into consideration, as there are sections with obsolete traffic principles on which $\mathrm{h}$ ]new designs are to follow which, in turn, will lead to supplementation of the design and envisage a new protection barrier where imposed by the new traffic designs.

\section{References}

1. J. Hristoski, A. Petrovski, R. Ristov, Preparation of design documentation on the level of a basic design on the replacement of the guardrail on the road of Corridor 10 in Republic of North Macedonia. 1, pp. 1-21 (2019)

2. Public Enterprise for State Roads, Technical instruction on the application of the vehicle retention system on the state roads of the Republic of North Macedonia, pp.2022 (2019)

3. EN 1317-1.Terminology and general criteria for test methods (2010)

4. EN 1317-2. Performance classes, impact test acceptance criteria and test methods for safety barriers including vehicle parapets (2010)

5. EN 1317-3. Performance classes, impact test acceptance criteria and test methods for crash cushions (2010)

6. EN 1317-2. Product requirements andevaluation of conformity for vehicle restraint systems (2008) 